\title{
Microlensing by Free-Floating Brown Dwarfs
}

\author{
Hans Zinnecker \\ Astrophysikalisches Institut Potsdam, An der Sternwarte 16, \\ 14482 Potsdam, Germany
}

\begin{abstract}
We propose a near-infrared microlensing survey of the central 2 degree field of the Galactic Center, in an attempt to estimate the surface density and mass distribution of distant free-floating brown dwarfs in the bulge and in the disk, acting as lenses of bright stars towards the Galactic Center. We estimate the probability (optical depth) of microlensing events to be $10^{-7}$ and the typical timescale (full-width) of the amplification lightcurve to be about 1 week. The necessary wide-field NIR survey technology should soon be available on UKIRT, CFHT, and with VISTA at ESO/Paranal.
\end{abstract}

\section{What is microlensing?}

Microlensing is gravitational lensing without resolving the split images. Stellar mass lenses are not nearly massive enough to generate split images, only a "lightcurve" of the microlensed stars due to the light amplification associated with the proper motion of the lens can be observed. The lightcurve is achromatic, i.e. the same for each wavelength, and also symmetric around its maximum, two characteristics which distinguish the microlensing effect from other light variations (e.g. RR Lyrae stars, Cepheids, or eclipsing binaries).

Microlensing occurs when the observer, the lens (in our case a brown dwarf), and the source (in our case a bright star in the Galactic Center or Bulge region) all lie nearly in the same line of sight. Microlensing was originally suggested by Paczynski (1986); see also the excellent review by Paczynski (1996).

\section{Why is microlensing useful in the brown dwarf business?}

As the equation below will show, microlensing can essentially give us an idea of the mass density of field brown dwarfs (or even free-floating giant planets) in distant parts of the Galaxy, such as in the Bulge, where star counts can no longer be performed. Microlensing can thus constrain the substellar initial mass function in places where we can no longer derive it from a local luminosity function (e.g. Mera et al. 1998, Peale 1998, Reid 2001, Zinnecker 2001).

In addition, microlensing can in principle (but not in practice) also yield the mass of the lens by measuring the full-width of the lightcurve. Basically the full-width scales with the square root of the mass of the lens. This is why brown dwarfs with their very low masses have interestingly short (1 week) timescales for the light amplification they generate (by moving across the straight line of sight 
between the observer and the source whose brightness is amplified). However, microlensing cannot determine the mass of the lens uniquely, as the width of the lightcurve also depends on other parameters, namely the precise proper motion of the lens in the plane of the sky and the exact distance to the lens and the source from the observer (see Paczynski 1998 for breaking this degeneracy).

\section{A crash course in microlensing (including basic equations)}

Three ingredients are needed to obtain a crude understanding of microlensing: the definition of the Einstein radius (roughly speaking the cross section), the shape of the light amplification curve as a function of time including the full-width of that light curve and its dependence on the lens mass and other parameters, and finally the expression for the probability, often called the optical depth, of microlensing.

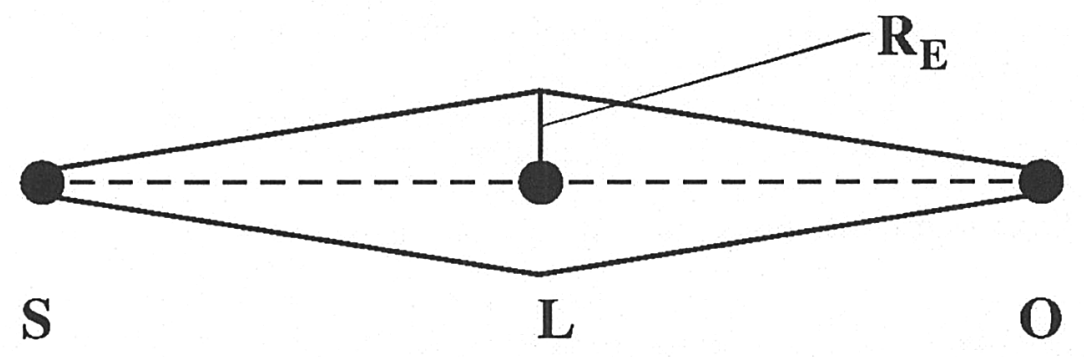

source

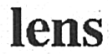

observer

Figure 1. Geometry of a gravitational microlensing situation

Fig. 1 shows the basic geometry for a microlensing situation where the lens happens to lie exactly in the $\mathrm{S}-\mathrm{O}$ line of sight. $R_{E}$ is the Einstein radius, defined as

$$
R_{E}=\sqrt{\frac{4 G M_{L}}{c^{2}} D_{S} x(1-x)}
$$

with $M_{L}$ the lens mass, $D_{s}$ the source distance from the observer, and $\mathrm{x}$ the ratio of lens distance to source distance. ( $G$ is the gravitational constant and $c$ is the speed of light). Putting numbers into the equation, we find

$$
R_{E}=1 A U
$$

for $M_{L}=0.03 M_{\odot}, D_{s}=10 k p c, x=0.5$, corresponding to a brown dwarf located halfway between us (the observer) and the Galactic Center, i.e. a bulgedisk lensing situation. If we want to have a bulge-bulge lensing situation ("selflensing"), we must choose $x=0.9$ and we obtain $R_{E}=0.6 \mathrm{AU}$. The most 
relevant $x$ for our purpose lies in between, as the expression $x(1-x)$ must be weighted with the mass density distribution of brown dwarfs as a function of $x$ (see below), which is higher for larger $x$, as the density of stars and likely for brown dwarfs increases towards the Galactic Center.

Next we have to define the impact parameter u (in units of $R_{E}$ )

$$
u=u(t)=\sqrt{u_{m i n}^{2}+\left[\frac{\left(t-t_{0}\right)}{2 t_{E}}\right]^{2}}
$$

where $u_{\min }=u\left(t_{0}\right)$ is the distance of closest approach (in units of $R_{E}$ ) at time $t_{0}$ of the moving lens to the line of sight between source and observer. For a closest approach equal to the Einstein radius $\left(u_{\min }=1\right)$, the source amplication factor $A$, given by (Paczynski 1986)

$$
A=\frac{1}{u} \frac{u^{2}+2}{\sqrt{u^{2}+4}}
$$

amounts to $A=1.34$. For a binary lens (i.e. a brown dwarf or a planet as companions to a low mass star lens), the impact parameter must be smaller than $u=1$, in order to get a bigger amplification and a better caustic crossing ( $u=0.1$ yields $A \approx 10$ ). This is needed to detect a deviation from a single lens lightcurve; see Mao \& Paczynski (1991), Gould \& Loeb (1992), Wambsganss (1997), and also Gaudi (these Proc.). Of course, such events are correspondingly rarer than $u=1$ events.

Next, we define the duration ( $\approx$ full-width at half maximum of $A=A(t)$ ) of the amplification light curve caused by the lens proper motion (relative tangential velocity). This is given by (Paczynski 1986)

$$
t_{E}=\frac{R_{E}}{v}
$$

where $v$ is the proper motion of the lens across the line of sight towards the source. For $R_{E}=0.6 \mathrm{AU}$ (see above) and $v=\sqrt{2} \cdot 100 \mathrm{~km} / \mathrm{s}$ (appropriate, given the Bulge velocity dispersion $\sim 100 \mathrm{~km} / \mathrm{s}$, similar for both sources and lenses), we find $t_{E} \approx 7$ days.

\section{The probability of microlensing events}

By measuring the distribution of the duration of a number of microlensing events one can estimate the probability for microlensing by brown dwarfs in the inner Galaxy at any given time. Given a surface number density of brown dwarfs towards the Galactic Center, the probability in question is basically given by the area coverage factor of all Einstein rings of the field brown dwarfs taken together, i.e.

$$
P=\pi R_{E, B D}^{2} N_{B D}
$$


where $N_{B D}$ is the total brown dwarf surface number density. Inserting the definition of $R_{E}$ and $x$, we can rewrite $P$ as

$$
\begin{aligned}
P & =\frac{4 \pi G D_{S}^{2}}{c^{2}} \int_{0}^{1} \rho(x) x(1-x) d x \\
& =\frac{2 \pi}{3} \frac{G \bar{\rho}_{B D}}{c^{2}} D_{S}^{2}
\end{aligned}
$$

Assuming $D_{s}=8-10 \mathrm{kpc}$ and replacing $\rho(x)$ by an average brown dwarf mass density $\bar{\rho}_{B D}=0.015-0.025 M_{\odot} / p c^{3}$ (3-5 times the local value in the solar neighborhood), we can evaluate the expression and obtain

$$
P \approx 10^{-7}
$$

Here we made the implicit assumption that the local brown dwarf number density is approximately equal to the number density of $\mathrm{M}$ dwarfs in the solar neighborhood (0.1stars $/ p c^{3}$, Reid 1999). We also took a representative brown dwarf mass of $0.05 M_{\odot}$. The rest is a scaling of the galactic radial density profile.

\section{From OGLE to IGLE: microlensing in the near-IR}

Microlensing in the near-IR K-band was originally proposed by Gould (1995). Not aware of this pioneering paper, the idea occurred to us to extend the optical gravitational microlensing experiment (OGLE) from Baade's Window $(l= \pm 1$, $b=-4$ in galactic coordinates) to a $\mathrm{K}$-band infrared microlensing survey (IGLE) of the central $2^{\circ} \times 2^{\circ}$ around the Galactic Centre, a region where optical studies are hopeless due to the high extinction $\left(A_{v} \leq 30 \mathrm{mag}\right)$; see Catchpole et al. (1990) and Mezger et al. (1999) for a K-band survey of the area, see also the 2MASS images.

The PI of OGLE is Prof. Udalski of Warsaw University whose team succeeded in detecting microlensing events towards the Galactic Bulge, thanks to a technique described as 'differential image analysis' (Alard \& Lupton 1998) with which time-variable sources, magnified by microlensing, could be convincingly recognized in large and very crowded CCD fields (Udalski et al. 2000, Wozniak et al. 2002). A similar technique should be applied to near-IR imaging surveys to beat the even higher crowding towards the central Galactic Bulge region.

\section{Bulge giants lensed by Bulge brown dwarfs}

By concentrating on the inner Galactic Bulge (Galactic Centre) region we maximise the number density of bright red giant sources to be lensed by faint brown dwarfs also in the Bulge (bulge-bulge lensing, see Kiraga \& Paczynski 1994). Red giants have an absolute K-magnitude close to $-3 \mathrm{mag}$, thus for a distance modulus $m-M=14.5 \mathrm{mag}$ to the Galactic Centre and an extinction of $A_{K}=3 \mathrm{mag}$ (corresponding to $A_{v}=30 \mathrm{mag}$ ) the apparent magnitude of red giants near the Galactic Centre becomes $K=14.5 \mathrm{mag}$. On a $4 \mathrm{~m}$ class telescope, a $\mathrm{S} / \mathrm{N}=20$ detection of a $10 \%$ brightness variation in sub-arcsec seeing takes about $2 \mathrm{~min}$, so 
near-IR surveys with $4 \mathrm{~m}$ class telescopes can proceed fast enough to observe an area of $2^{\circ} \times 2^{\circ}$ several times a day for several days, enough to catch microlensing events of short duration (few days); such a survey will have other useful spin-offs (e.g. probing galactic bar structure: see Peale, 1998; Evans \& Belokurov, 2002).

\section{Telescopes/instruments available}

For the purposes of the proposed near-IR microlensing survey towards obscured central Galactic Bulge sources, there are practically 4 choices of telescopes and instrumentation:

- the wide-field camera WFCAM for UKIDSS at UKIRT (Warren 2002), ready for use in 2004 , with a $2 \times 2$ arrangement of $2 \mathrm{k} \times 2 \mathrm{k}$ detectors at $0.4^{\prime \prime} /$ pix (total instantaneous FOV per exposure $\approx 0.2 \square^{\circ}$ )

- the wide-field camera WIRCAM at CFHT (Beuzit, these Proc.), ready for use in 2004, with a total instantaneous FOV per exposure of about $0.1 \square^{\circ}$ $\left(4 \mathrm{k} \times 4 \mathrm{k}=20^{\prime} \times 20^{\prime}\right)$ at $0.3^{\prime \prime} / p i x$

- the planned $1 \square^{\circ}$ FOV telescope VISTA at ESO/Paranal, to be ready in 2006 , with a $4 \times 4$ arrangement of $2 \mathrm{k} \times 2 \mathrm{k}$ near-IR detectors at $0.34 " / p i x$ (practically a $2 \times 2$ extension of WFCAM for UKIDSS at UKIRT)

- the NGST near-infrared camera, planned for 2010 , with two $4 \mathrm{k} \times 4 \mathrm{k}$ detector at $0.04 " / p i x$ in the K-band, i.e. a total FOV of about $3^{\prime} \times 3$ '.

The two immediate options (at UKIRT/CFHT) each provide some 16 million pixels per exposure, while the two medium-term possibilities (VISTA/NGST) feature 64 and 48 million pixels. In order to cover the $2^{\circ} \times 2^{\circ}$ Galactic Centre area, we need about 20, 40, and 4 exposures with WFCAM, WIRCAM, and VISTA, respectively. In each of these cases ${ }^{1}$, we can sample on the order of $3 \cdot 10^{8}$ pixels and complete the high $\mathrm{S} / \mathrm{N}$ observations of the bright bulge giants within $1 \mathrm{hr}$ (integration plus read-out time). We suggest 3 sets of observations per night for 14 consecutive nights. This should be good enough to probe the previously estimated probability of $10^{-7}$ for microlensing by bulge brown dwarfs.

\section{Acknowledgment}

Travel support from the Deutsche Forschungsgemeinschaft (DFG) is gratefully acknowledged. I also thank Bogdan Paczynski, Penny Sackett, Robert Schmidt and Joachim Wambsganss for stimulating discussions.

\footnotetext{
${ }^{1}$ NGST is different. It will not observe a large FOV, but will operate mostly in staring mode and will go much deeper. The hope is to resolve Bulge subgiants and Main Sequence stars.
} 


\section{References}

Alard, C. \& Lupton, R. H. 1998, ApJ 503, 325

Catchpole, R. M., Whitelock, P., \& Glass, I. S. 1990, MNRAS 247, 479

Delplancke, F., Gorski, K. M., \& Richichi, A. 2001, A\&A 375, 701

Evans, N. W. \& Belokurov, V. 2002, ApJ 567, L119

Gould, A. 1995, ApJ 446, L71

Gould, A. \& Loeb, A. 1992, ApJ 396, 104

Kiraga, M. \& Paczynski, B. 1994, ApJ 430, L101

Mao, S. \& Paczynski, B. 1991, ApJ 374, L37

Mera, D., Chabrier, G., \& Schaeffer, R. 1998, A\&A 330, 937

Mezger, P. G., Zylka, R., Philipp, S., \& Launhardt, R. 1999, A\&A 348, 457

Paczynski, B. 1986, ApJ 304, 1

Paczynski, B. 1996, ARA\&A 34, 419

Paczynski, B. 1998, ApJ 494, L23

Peale, S. J. 1998, ApJ 509, 177

Reid, I. N., Kirkpatrick, J. D., Liebert, J. et al. 1999, ApJ 521, 613

Reid, I. N. 2001 in ASP Conference Proceedings Vol. 239,

Microlensing 2000: A New Era of Microlensing Astrophysics, ed. J. W. Menzies \& P. D. Sackett (San Francisco: ASP), 327

Udalski, A., Zebrun, K., Szymanski, M. et al. 2000, Acta Astronomica 50, 1

Wambsganss, J. 1997, MNRAS 284, 172

Warren, S. 2002, The Messenger 108, 31

Wozniak, P. R., Udalski, A., Szymanski, M. et al. 2002, Acta Astronomica 52, 129

Zinnecker, H. 2001 in ASP Conference Proceedings Vol. 239, Microlensing 2000: A New Era of Microlensing Astrophysics, ed. J. W. Menzies \& P. D. Sackett (San Francisco: ASP), 223 (and astro-ph 0107351)

\section{Note added in proof}

Delplancke et al. (2001) discussed the possibilities to resolve the images of microlensed objects towards the Galactic Bulge by the use of long baseline interferometry, in particular the ESO-VLTI (K-band observations with the AMBER and PRIMA instruments). The ability to measure the angular separation between the microlensed images (of the order of 1 milli-arcsec) and to measure the brightness of the images as a function of time will enable a direct and unambiguous determination of the lens masses and locations, as well as their proper motions. This then allows one to break the degeneracy (mass, tangential velocity, distance of the lens) inherent in the interpretation of the width of the lightcurve of a microlensing event. 